\title{
Sialorrea neurogénica infantil y el manejo con la toxina Botulínica: revisión de la literatura y reporte del caso de un niño con traqueostomía y ventilación mecánica crónica
}

\author{
Dr. S. Chahuán', Dra. Tatiana Espinoza-Palma², Dr. L. Cruzat ${ }^{4}$, D. Saumann ${ }^{5}$ Dra. L. Berna ${ }^{3}$ \\ 1 Médico Fisiatra, Instituto Nacional de Rehabilitación Pedro Aguirre Cerda. \\ 2 Médico Broncopulmonar infantil, Instituto Nacional de Rehabilitación Pedro Aguirre Cerda. \\ 3 Médico Fisiatra, Instituto Nacional de Rehabilitación Pedro Aguirre Cerda. \\ 4 Pediatra, Instituto Nacional de Rehabilitación Pedro Aguirre Cerda. \\ 5 Fonoaudióloga, Instituto Nacional de Rehabilitación Pedro Aguirre Cerda.
}

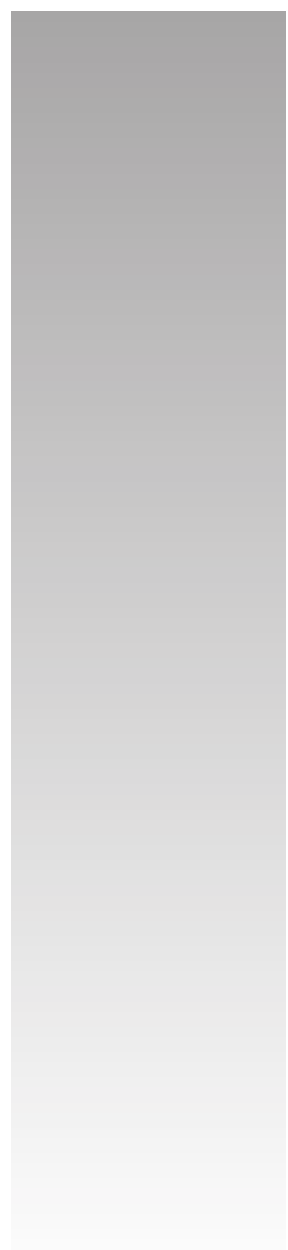

\begin{abstract}
NEUROGENIC DROOLING CHILD AND HIS TREATMENT WITH BOTULINUM TOXIN: A REVIEW OF THE LITERATURE AND CASE REPORT OF A CHILD WITH CHRONIC TRACHEOSTOMY AND MECHANICAL VENTILATION
\end{abstract}

Drooling is defined as non-intentional salivary loss from mouth. Its presence may lead to functional, psychological and social impairments, which affect patients and caregivers as well. Anterior drooling, where salivary flow escapes from mouth to outside, affects social relations, constituting a physical contact barrier and having a negative impact on quality of life, and it also interfere on speech and communication. Posterior drooling, where salivary flow goes towards the throat is associated to severe swallowing disorders, being an important aspiration risk. Although drooling etiology is multifactorial, it's often seen associated to a neuromuscular dysfunction. The approach to drooling should be made by a multidisciplinary team. Therapeutic approach could be non-pharmacologic, pharmacologic, systemic or local. Ultrasound guidance Botulinum toxin injection is an efficient and safe alternative. A 4-year-old male with posterior fossa astrocytoma, VI-XI CN impairment, prolonged mechanical ventilation and tracheostomy, swallowing disorder and hypersalivation is presented. Drooling management with botulinum toxin A injection in both parotid and submandibular glands under ultrasound guidance was performed, with an important salivary flow decrease, which allowed him to open his mouth without saliva loss, having a positive impact on communication and socialization, improving his quality of life as well.

Key words: Drooling, onabotulinumtoxin A, tracheostomy.

\section{RESUMEN}

La sialorrea se define como la pérdida no intencional de saliva desde la boca. Su presencia puede conducir a alteraciones funcionales, psicológicas y sociales, que afectan tanto al paciente como a sus cuidadores. La sialorrea anterior, donde la saliva es derramada desde la boca al exterior afecta la socialización, constituyéndose como una barrera para el contacto físico e impacta en forma negativa sobre la calidad de vida, además interfiere en el lenguaje, el habla y la comunicación. La sialorrea posterior que es derramada hacia del itsmo de las fauces, se asocia a trastornos severos de la deglución, constituyendo un riesgo importante de aspiración. Aún cuando la etiología de la sialorrea es multifactorial, frecuentemente la vemos asociada a una disfunción neuromuscular. Su abordaje debe ser realizado por un equipo multidisciplinario. El enfoque terapéutico puede ser, no farmacológico o farmacológico, sistémico o local. Una de las alternativas es la infiltración con toxina botulínica guiada en forma ecográfica que ha demostrado ser eficiente y segura. Se presenta el caso de un preescolar de 4 años con antecedente de astrocitoma de fosa posterior, compromiso de $\mathrm{VI}$ al XI pares craneanos, ventilación mecánica prolongada a través de traqueostomía, trastorno de deglución e hipersalivación secundaria. Se realiza el manejo de la sialorrea con toxina botulínica A en parótidas y glándulas submandibulares con marcación ecográfica, resultando una disminución importante del flujo de saliva que le permite abrir la boca sin escurrimiento. Lo anterior impacta positivamente en la comunicación y socialización, además de mejorar su calidad de vida.

Palabras clave: Sialorrea, onabotulinumtoxin A, traqueostomía.

Correspondencia: Dra. Tatiana Espinoza. Pediatra Broncopulmonar.

Instituto Nacional de Rehabilitación Pedro Aguirre Cerda.

E-mail: tatita25@gmail.com 


\section{INTRODUCCIÓN}

La sialorrea se define como la pérdida no intencional de saliva desde la boca ${ }^{(1)}$. Esta condición es normal en los niños pequeños hasta los 15 ó 18 meses de edad, pero se considera una entidad patológica cuando ocurre después de los cuatro años ${ }^{(2-5)}$. Su presencia puede conducir a alteraciones funcionales, psicológicas y sociales, que afectan tanto al paciente como a su familia o cuidadores. Uno de los problemas más importantes es que impide la socialización, entorpece las relaciones interpersonales y la integración en la sociedad, constituyéndose como una barrera para el contacto físico(l).

Con respecto a la etiología y al impacto clínico es importante distinguir la sialorrea anterior de la sialorrea posterior. La sialorrea anterior se refiere a la saliva derramada desde la boca, que es claramente visible. La sialorrea posterior es cuando la saliva es derramada hacia el istmo de las fauces, creando un riesgo de aspiración. La sialorrea posterior ocurre en pacientes con disfagia orofaringea severa. Ambas se pueden encontrar en un mismo paciente ${ }^{(1)}$. Existen múltiples estudios que muestran la experiencia del manejo de la sialorrea con la infiltración de toxina botulínica en niños con alteraciones neurológicas como parálisis cerebral. Sin embargo, no existen estudios en la literatura nacional ni internacional sobre el uso de la infiltración de toxina botulínica en niños con traqueostomía dependiente de ventilación mecánica.

El objetivo de este trabajo es presentar una revisión de la literatura sobre la sialorrea con su manejo principalmente a través de toxina botulínica y compartir la evolución de un paciente con traqueostomía dependiente de ventilación mecánica a quien se manejo la sialorrea con infiltración de toxina botulínica A en glándulas salivales submandibulares y parótidas, marcadas por ecografía.

\section{REVISIÓN DE LA LITERATURA}

La secreción de saliva es regulada a través de un arco reflejo. La vía aferente es activada por la estimulación de quimioreceptores y mecanoreceptores. Los nervios craneales V, VII, IX y $\mathbf{X}$, llevan los impulsos hacia el centro de la salivación que se encuentra en protuberancia y bulbo raquídeo. La vía eferente de este arco reflejo es principalmente parasimpática. EI VII par craneal entrega el control a las glándulas submandibulares, sublingual y glándulas menores, mientras que el IX par craneal controla la salivación de las glándulas parótidas. El flujo de saliva es reforzado por la inervación simpática, la cual promueve la contracción de las fibras musculares que rodean los conductos salivales².

En estado de reposo, el 70\% de la saliva es secretada por las glándulas submandibulares y sublinguales. Bajo estimulación, el flujo de saliva aumenta hasta cinco veces, siendo la glándula parótida el principal proveedor. Un adulto produce entre $1.000-1.500 \mathrm{ml}$ de saliva por día, los niños antes de la pubertad producen significativamente menos $(750-900 \mathrm{ml}$ por día) $)^{(1)}$.

La saliva cumple varias funciones, entre las que se cuentan la limpieza mecánica de la boca, contribuir a la homeostasis oral y a regular el $\mathrm{pH}$ de la boca. La saliva también tiene
Tabla I. Etiología de la sialorrea

\begin{tabular}{|l|}
\hline Disfunción neuromuscular/sensorial \\
Retardo mental \\
Parálisis cerebral \\
Enfermedad de Parkinson \\
Parálisis pseudobulbar \\
Parálisis bulbar \\
Accidente cerebrovascular \\
Hipersecreción \\
Inflamación \\
Medicamentos (tranquilizantes, anticonvulsivantes) \\
RGE \\
Exposición a tóxicos (mercurio) \\
Anatómicas \\
Macroglosia \\
Incompetencia oral \\
Malaoclusión dental \\
Problemas de ortodoncia \\
Defectos quirúrgicos de cabeza y cuello \\
\end{tabular}

propiedades bacteriostáticas y bactericidas que contribuyen a la salud dental y a disminuir el mal olor. Es importante en la lubricación del bolo alimenticio y la amilasa que contiene inicia la digestión de los hidratos de carbono ${ }^{(5-8)}$. En un ciclo de 24 horas ocurren alrededor de 600 degluciones de saliva. En condiciones normales un individuo es capaz de compensar un aumento de salivación, por lo que, en general, sólo una hipersalivación no produce sialorrea, al contrario una alteración de la deglución casi siempre la provoca(2).

Si bien es correcto decir que la etiología de la sialorrea es multifactorial, podríamos dividir sus causas en 3 grandes grupos: la causada por disfunción neuromuscular y sensorial, hipersalivación y alteraciones anatómicas (Tabla I). Es ampliamente aceptado que la sialorrea en pacientes con parálisis cerebral infantil $(\mathrm{PCl})$, no es causada por hipersalivación, sino más bien por un mal control motor oral. Una excepción a esto pueden ser los desórdenes diskinéticos, donde los movimientos anormales pueden estimular constantemente a la glándula parótida y producir más saliva ${ }^{(1)}$.

La prevalencia reportada para la sialorrea en los diferentes estudios varía para la $\mathrm{PCl}$ entre 10 y $38 \%(1,2,7,8)$. Incluso hay algunos estudios que han reportado que en $50 \%$ de los pacientes con $\mathrm{PCl}$ han presentado algún grado de sialorrea en algún momento de su evolución. En el 10\% de estos pacientes se producirían importantes alteraciones en actividades sociales y funcionales. Otros estudios han mostrado prevalencias cercanas al $60 \%$ en niños en escuelas especiales, de las cuales el 33\% podría clasificarse como severa ${ }^{(7,9)}$.

La morbilidad asociada a la sialorrea es muy variada y de diferente impacto. Los niños con sialorrea severa muchas veces presentan maceración de la piel, infecciones alrededor de la boca y barbilla, mayor presencia de caries dentales, alteraciones en la dentición y deglución, incluso si la pérdida de fluidos es importante, ésta puede llevar a deshidratación ${ }^{2,7}$. También existe el riesgo de neumonía aspirativa en casos de asociación a disfagia ${ }^{(9-1)}$.

La presencia de sialorrea también tiene un impacto en 
el ámbito psicológico y social, muchas veces es motivo de vergüenza, restringe el contacto físico directo y lleva a aislamiento social(9). Todas estas complicaciones llevan por una parte a incrementar los costos en salud y por otra parte, a disminuir la calidad de vida del paciente y su familia ${ }^{(3)}$. Como la sialorrea es un problema multifactorial, requiere idealmente de la valoración por un equipo multidisciplinario!

La entrevista inicial debería incluir una completa historia médica, social y emocional del paciente. La evaluación por fonoaudiólogo es esencial, esta debería incluir una evaluación de los órganos fonoarticulatorios (función y anatomía), sensibilidad, reflejos, evaluación postural, del mecanismo de deglución y de la deglución de saliva propiamente tal. El examen dental también es recomendado en los mayores de 3 años, las caries dentales y la pobre higiene dental puede contribuir a una excesiva secreción de saliva. El cuantificar en forma objetiva el flujo de saliva es una tarea difícil, por lo que se han descrito distintos métodos en los diferentes estudios. Dentro de los más utilizados están las tórulas dentales, aspiración de saliva y más recientemente las micropipetas para canulación retrógrada de los conductos de las glándulas salivales mayores ${ }^{(1)}$.

Existen también escalas para cuantificar en forma subjetiva el flujo de saliva, la más usada es la escala de severidad y frecuencia de la sialorrea (Tabla 2) $)^{(1,5)}$.

El impacto de la sialorrea en la calidad de vida es el factor más importante para determinar la necesidad de terapia en el caso de sialorrea anterior ${ }^{(5)}$. A este respecto existe una herramienta que ha sido recientemente validada que evalúa el impacto de la sialorrea en la calidad de vida del paciente y sus cuidadores, es la escala de impacto de la sialorrea ${ }^{(1,4)}$. Ésta consta de 10 preguntas, cada una con rango de puntaje a otorgar de I a I0. Esta evaluación refleja los cambios en la sialorrea percibidos por la persona que contesta el cuestionario. Las opciones de tratamiento de la sialorrea pueden ser agrupadas en 4 categorías:

I. Manejo funcional.

2. Medicamentos orales.

3. Infiltración intraglandular de toxina botulínica.

4. Manejo quirúrgico.

El manejo funcional incluye la instrucción repetitiva, el refuerzo positivo o negativo, la estimulación de la deglución segura o trabajar sobre sus componentes alterados (sensibilidad, control motor oral, etc.) y la autocorrección. Al respecto, la evidencia es limitada, pero existe consenso en que es beneficiosa cuando se usa como terapia complementaria a alguna de las otras opciones de tratamiento(12).

Dentro de los medicamentos más utilizados están los anticolinérgicos (escopolamina, glicopirrolato), que bloquean la inervación parasimpática a las glándulas salivales. La evidencia en cuanto a su efectividad es insuficiente, lo que sí es claro es que la presencia de efectos secundarios es alto (44-66\%). Dentro de los más frecuentes se encuentran la sedación, constipación, confusión y retención urinaria(5, 13-14).

Las opciones de tratamiento quirúrgico incluyen la escisión glandular, la reubicación del conducto, ligadura del conducto y neurotomías. Estas técnicas, si bien se ha reportado que serían altamente efectivas, son invasivas e irreversibles,
Tabla 2. Escala de severidad y frecuencia de sialorrea

\begin{tabular}{|lc|}
\hline Sialorrea & Puntos \\
\hline Severidad & \\
Seco & 1 \\
Leve (moja los labios) & 2 \\
Moderada (moja labios y barbilla) & 3 \\
Severa (moja ropa) & 4 \\
Profusa (moja ropa, manos, objetos) & 5 \\
Frecuencia & \\
Nunca & 1 \\
Ocasionalmente & 2 \\
Frecuentemente & 3 \\
Constantemente & 4 \\
\hline
\end{tabular}

por lo que sólo se deben considerar en sialorreas severas en las que las otras alternativas de tratamiento no tuvieron los resultados positivos ${ }^{(2,5-6)}$.

Finalmente, se describe el manejo con toxina botulínica en glándulas salivales. Esta actúa inhibiendo la liberación de acetilcolina desde la terminal nerviosa colinérgica, produciendo de este modo una especie de denervación química, reduciendo la secreción de saliva y la sialorrea en la mayoría de los pacientes. Sus beneficios y relativa seguridad han sido ampliamente documentados en varios estudios ${ }^{(3,5)}$. Varios autores han demostrado la reducción clínicamente significativa del flujo de saliva en 33-64\% de los pacientes por aproximadamente 2 a 6 meses $^{(6)}$.

La mayoría de los estudios son para el serotipo A de toxina botulínica; sin embargo, la eficacia del serotipo B también ha sido probada con similares resultados en niños con parálisis cerebral y enfermedades neurodegenerativas en un número más reducido de estudios ${ }^{(1)}$. La evidencia en general nos dice que la infiltración con toxina en glándulas salivales es segura y efectiva en el tratamiento de la sialorrea y es más eficiente cuando se infiltran ambas glándulas, parótida y submandibular ${ }^{(1,10)}$. Todavía es necesario definir mejor las dosis a utilizar, en la literatura se describe un amplio rango de variación en las dosis (Tabla 3).

En cuanto a los efectos adversos de la infiltración con toxina botulínica, estos se pueden minimizar si el procedimiento es llevado a cabo con la adecuada sedación del paciente, de modo que se encuentre quieto durante la infiltración y usando la guía ecográfica.

No se han reportado efectos fatales en la literatura, pero sí varios potenciales efectos colaterales, que deberían ser cuidadosamente monitorizados. Estos efectos los podemos

Tabla 3. Sitio de infiltración y dosis

\begin{tabular}{|lcc|}
\hline & $\begin{array}{c}\text { Botox R (U) } \\
\text { Toxina } \\
\text { botulínica A }\end{array}$ & $\begin{array}{c}\text { Dysport R (U) } \\
\text { Toxina } \\
\text { botulínica A }\end{array}$ \\
\hline Glándula submandibular & $10-50$ & $15-75$ \\
Glándula parótida & $10-50$ & $15-75$ \\
\hline
\end{tabular}


dividir en aquellos derivados del trauma en el sitio de infiltración y los relacionados a la toxina botulínica. En el primer grupo destaca el dolor en el sitio de infiltración, formación de hematoma, inflamación glandular, infección y posibilidad de trauma del nervio facial. En el segundo grupo encontramos, problemas del manejo intraoral de alimentos sólidos por excesiva sequedad de la boca, espesamiento de la saliva, trastorno de deglución por difusión de toxina a musculatura vecina, debilidad en la masticación por difusión a masétero y pérdida de toxina por inyección intravascular (sin consecuencias sistémicas en general por las bajas dosis usadas, sin embargo, el botulismo iatrogénico ha sido descrito en un caso)(').

\section{CASO CLÍNICO}

Se presenta el caso de un paciente preescolar de 4 años, con antecedente de astrocitoma de fosa posterior operado (marzo de 20 I I), proveniente del Hospital Roberto del Río, originario de Vallenar, hospitalizado en la Unidad de Cuidados Especiales (UCE) del Instituto Nacional de Rehabilitación Pedro Aguirre Cerda (INRPAC) para rehabilitación integral. Presentaba las siguientes secuelas: hidrocefalia comunicante con válvula DVP, compromiso del VI al XI par craneal y de tronco encefálico, tetraparesia fláccida, insuficiencia respiratoria crónica manejado con ventilación mecánica prolongada a través de traqueostomía, disfagia con gastrostomía, vaciamiento gástrico lento y reflujo gastro-esofágico patológico. Al examen físico se evidenció un niño muy conectado al medio y colaborador. Presentaba una fascie hipomímica; no abría la boca por presentar acúmulo de saliva en la cavidad oral, secundaria a su disfagia. Al estar conectado al medio y cognitivamente consciente de su dificultad, evitaba abrir la boca. Presentaba una parálisis de cuerdas vocales en abducción, por lo que uno de los principales mecanismos de protección de la vía aérea se encontraba alterado. Al intentar tragar la saliva no se activaban ni el reflejo de deglución ni los reflejos protectores, por lo que todo el volumen pasaba directamente a tráquea. Por otro lado, tenía pobre control de tronco y no lograba marcha. Se inició una intervención por el equipo de rehabilitación del INRPAC compuesto por pediatra, fisiatra, neurólogo, kinesiólogo, fonoaudiólogo, terapeuta ocupacional y educadora diferencial.

Evolucionó en forma favorable, logrando mayor estabilidad postural, mejor coordinación y recuperó la marcha. Sin embargo, persistía sin querer abrir la boca por el escurrimiento salival. Se recolectaban diariamente I.000 cc de saliva a través de aspiración casi continua que era manejada por el propio paciente. Se decidió suspender las aspiraciones por ser un estímulo para la producción de saliva. Al recolectar la saliva en vaso disminuyó el volumen a 800 cc/día, lo cual es acorde a la producción de la misma esperable para su edad.

Se evaluaron distintas alternativas de tratamiento farmacológico, pero todas eran de efecto transitorio y con graves secuelas adversas dada su condición de vaciamiento gástrico enlentecido con RGE patológico, lo que aumentaba el riesgo de aspiración. Se optó por realizar la infiltración de toxina botulínica A en glándulas submandibulares y parótidas. Los objetivos que nos propusimos con la infiltración fueron disminuir el riesgo de aspiración, favorecer la intención de comunicación y facilitar el habla a través del entrenamiento con válvula de fonación. A partir de lo anterior también se favoreció una deglución segura al estimular las cuerdas vocales como mecanismo de protección de la vía aérea, mejorar la sociabilización y su calidad de vida. Previo al procedimiento se realizó medición objetiva y subjetiva del flujo de saliva aplicando la escala de severidad y frecuencia de la sialorrea y la escala de impacto de la sialorrea (EIS). Se efectuó marcación de los cuerpos glandulares a través de ecografía en el Servicio de Radiología Infantil del Hospital Sótero del Río y posteriormente el médico fisiatra infiltró al paciente en la UCE bajo sedación, de acuerdo a pauta preestablecida, con un total de $70 \cup$ de onabotulinumtoxin A (Figura I).

Los resultados se midieron a las $24 \mathrm{hrs}$, a las semanas I, $2,4,8,16$. Se apreció una franca disminución del volumen de saliva a las 24 h post infiltración, la que es máxima a las dos semanas y se pierde parcialmente a los 5 meses (Figura 2). Además, se pudo apreciar disminución en las escalas de severidad y frecuencia y EIS que ha persistido en el tiempo (Figuras 3 y 4). Logró abrir la boca sin que ocurriera escurrimiento de saliva y se comenzó a estimular la fonación, logrando emitir sonidos con voz disfónica.
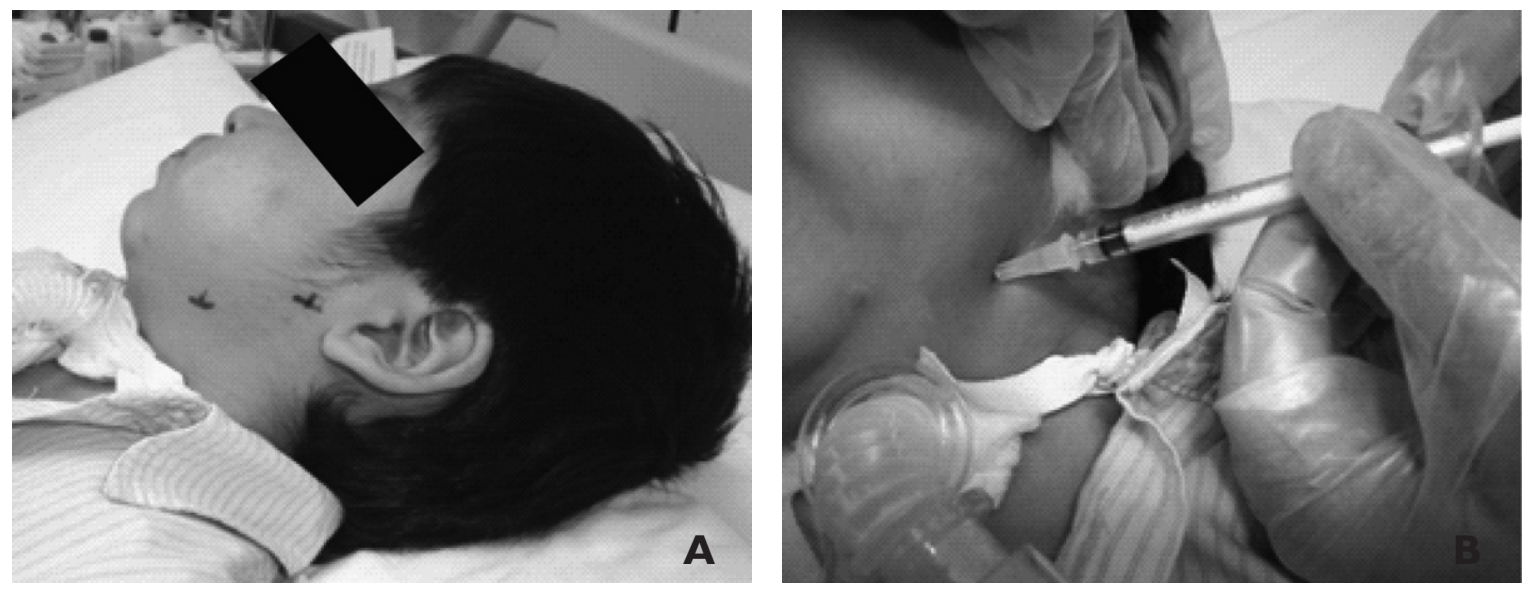

Figura I. 


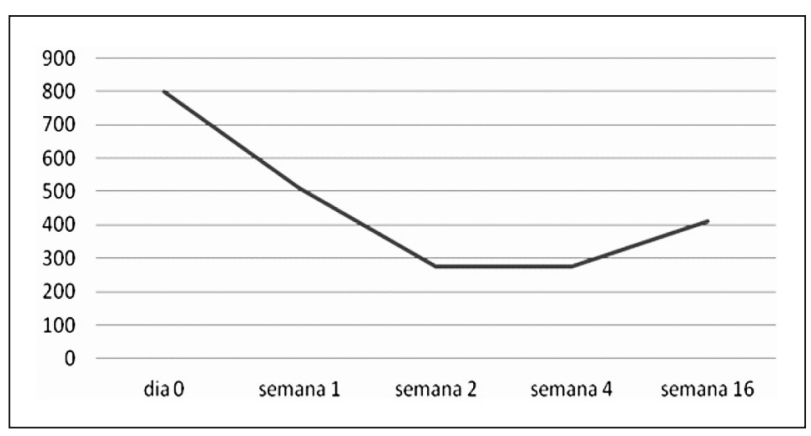

Figura 2. Cantidad de saliva recolectada en 24 horas.

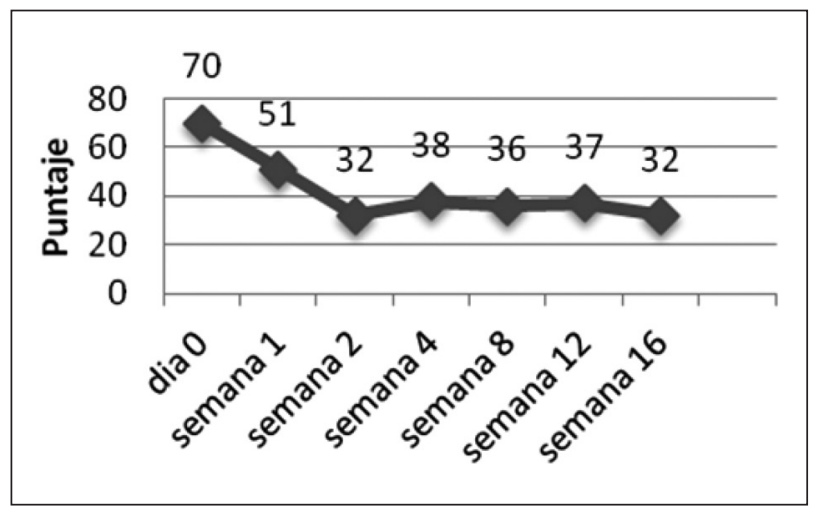

Figura 3. Escala de impacto de sialorrea.

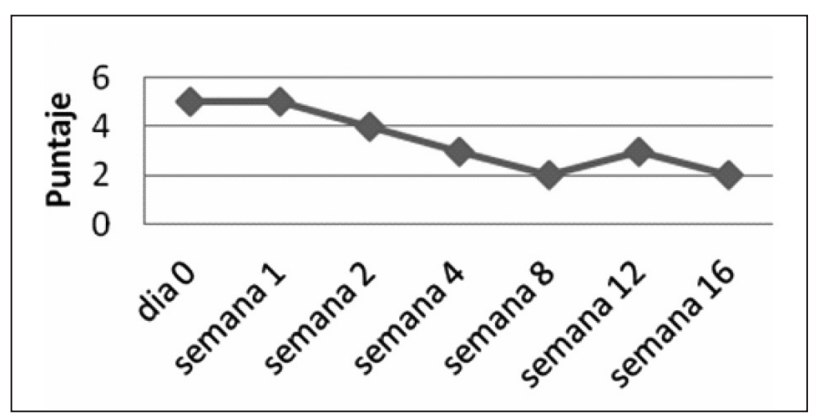

Figura 4. Severidad de la sialorrea.

\section{DISCUSIÓN}

El caso presentado corresponde al primero documentado del uso de toxina botulínica en glándulas salivales en un paciente con ventilación mecánica prolongada a través de traqueostomía y disfagia severa con gastrostomía para el manejo de la sialorrea. Se decidió realizar este procedimiento pese a los potenciales efectos adversos, como la aspiración por empeoramiento de la deglución('), porque contábamos con una vía de alimentación segura a través de la gastrostomía y además nos apoyamos efectuando la infiltración con marcación ecográfica de las glándulas salivales, lo que ha demostrado disminuir el riesgo de disfagia secundaria a la difusión de la toxina botulínica hacia músculos vecinos involucrados en la deglución ${ }^{(1,10)}$. Si bien solamente es un caso, no observamos efectos adversos.

Nuestro principal objetivo al tratar la sialorrea fue facilitar el lenguaje, el habla y la comunicación y con ello mejorar la socialización, pero al manejar menores volúmenes de saliva en la boca, se pudo iniciar la intervención sobre el trastorno de deglución, estimulando las cuerdas vocales como mecanismo de protección para una deglución segura. De esta manera logramos un impacto positivo sobre la calidad de vida.

Existen algunos casos descritos en la literatura de infiltración de toxina botulínica en pacientes con algunas características similares a nuestro paciente. Winterholler utilizó toxina botulínica en glándulas parótidas y submandibulares en un paciente con traqueostomía ${ }^{(15)}$. No se describió entonces efectos colaterales. Por otro lado, Ellies y colaboradores describieron la infiltración en un paciente con ventilación mecánica prolongada no invasiva ${ }^{(16)}$.

Actualmente, el niño está en su ciudad de origen, a cargo de su familia, insertado a la comunidad y apoyado por el Programa Nacional Ministerial de Ventilación Mecánica Invasiva $(\mathrm{AVI})$. La madre manifestó su deseo de que sea nuevamente sometido al procedimiento, debido a que la sialorrea está volviendo a su condición basal. Este es uno de los inconvenientes del tratamiento con toxina botulínica, ya que debe ser repetido en el tiempo ${ }^{(17)}$ pues la duración del efecto se prolonga por un máximo de 6 meses, como ocurrió con nuestro paciente. Se han descrito variaciones en la duración de tratamiento entre 6 semanas y 6 meses $^{(2)}$, otros estudios reportan un promedio de duración del tratamiento de 22 semanas $^{(6)}$.

Sin embargo, recientemente se ha documentado un efecto similar a la atrofia glandular, producido tras la infiltración repetida de toxina botulínica a ese nivel; se explicaría por la denervación química prolongada a la que las glándulas son expuestas. Por lo tanto, la sialorrea sería cada vez de menor cuantía y la necesidad de tratamiento sería cada vez menor y más espaciada en el tiempo. Esta observación requiere de una mayor cantidad de estudios a largo plazo.

Finalmente, es importante reforzar la importancia de realizar una intervención a través de un equipo multidisciplinario, que pueda dar un enfoque integral al manejo del paciente y sea capaz de determinar todas las áreas en las que impacta la sialorrea y abordarla en forma eficiente.

\section{CONCLUSIÓN}

La infiltración con toxina botulínica $A$, es una herramienta útil a considerar en pacientes portadores de sialorrea severa con traqueostomía y ventilación mecánica asociado a disfagia con vía de alimentación segura.

\section{REFERENCIAS}

I. Reddihough D, Erasmus CE, Johnson H, McKellar GMW, PH. J. Botulinum toxin assessment, intervention and aftercare for paediatric and adult drooling: international consensus statement. Eur J Neurol 2010; 17 (suppl 2): 109-21. 
2. Scully C, Limeres J, Gleeson M, I. T, P. D. Drooling. J. Oral Pathol Med 2009; 38: 321-7.

3. Meece RW, Fishlock KF, Bayley EW, Keller MS. Ultrasound-Guided Botox Injections of Salivary Glands in Children with Drooling. J Radiol Nursing 20 10; 29: 20-4.

4. Reid SM, Johnson HM, Reddihough DS. The Drooling Impact Scale: a measure of the impact of drooling in the children with developmental disabilities. Develop Med \& Child Neurol 2010; 52: e23-8.

5. Hockstein N, Samadi D, Gendron K, Handler S. Sialorrhea: A Management Challenge. Am Fam Physic 2004; 69: 2628-34.

6. Erasmus C, Van Hulst K, Rotteveel L, et al. Drooling in cerebral palsy: Hypersalivation or dysfunctional oral motor control? Develop Med \& Child Neurol 2009; 51 : 454-9.

7. Banerjee K, Glasson C, O'Flaherty S. Parotid and submandibular botulinum toxin A injections for sialorrhoea in children with cerebral palsy. Develop Med \& Child Neurol 2006; 48: 883-7.

8. van der Burg J, Jongerius P, van Hulst K, van Limbeek J, Rotteveel J. Droling in children with cerebral palsy: effect of salivary flow reduction on daily life and care. Develop Med \& Child Neurol 2006; 48: 103-7.

9. Scheffer A, Erasmus C, van Hulst K, van Limbeek J, Jongerius P, van den Hoogen F. Efficacy and Duration of Botulinum Toxin Treatment for Drooling in 131 Children. Arch Otolaryngol Head Neck Surg 2010; 136: 873-7.

10. Benson J, Daugherty K. Botulinum Toxin A in the Treatment of Sialorrhea. The Ann Pharma 2007; 41: 79-85.

I I. Laing TA, Laing ME, O'Sullivan ST. Botulinum Toxin for treatment of glandular hypersecretory disorders. J Plastic Reconstr \& Anesth Surg 2008; 61: 1024-8.

12. Van der Burg J, Didden R, Jongerius P, Rotteveel J. A descriptive analysis of studies on behavioural treatment of drooling (19702005). Develop Med \& Child Neurol 2007; 49: 390-4.

13. Bachrach S, Walter R, Trzcinski K. Use of glycopyrrolate and other anticholinergic medications for sialorrhea in children with cerebral palsy. Clin Pediatrics 1998; 37: 485-90.

14. Jongerius P, van Tiel P, van Limbeek J, Gabreels FJ, Rotteveel J. A systematic review for evidence of efficacy of anticholinergic drugs to treat drooling. Arch Dis Child 2003; 10: 91।-4.

15. Winterholler $M$. Treatment of sialorrhea in patients under longterm ventilation. Pneumologie 2008; 62(Suppl I): S39-42.

16. Ellies M, Laskawi R, Rohrbach-Volland S, Arglebe C, Beuche W. Botulinum toxin to reduce saliva flow: selected indications for ultrasound-guided toxin application into salivary glands. Laryngoscope 2002; I 12:82-6.

17. Little S, Kubba H, Hussain S. An evidence-based approach to the child who drools saliva. Clin Otolaryngol 2009; 34: 236-9. 